\title{
The dynamical origin of the multi-planetary system HD 45364
}

\author{
H. Rein ${ }^{1}$, J. C. B. Papaloizou ${ }^{1}$, and W. Kley ${ }^{2}$ \\ 1 University of Cambridge, Department of Applied Mathematics and Theoretical Physics, Centre for Mathematical Sciences, \\ Wilberforce Road, Cambridge CB3 0WA, UK \\ e-mail: hr260@cam.ac.uk \\ ${ }^{2}$ University of Tübingen, Institute for Astronomy and Astrophysics, Auf der Morgenstelle 10, 72076 Tübingen, Germany
}

Received 30 August 2009 / Accepted 26 October 2009

\begin{abstract}
The recently discovered planetary system HD 45364, which consists of a Jupiter and Saturn-mass planet, is very likely in a 3:2 mean motion resonance. The standard scenario for forming planetary commensurabilities is convergent migration of two planets embedded in a protoplanetary disc. When the planets are initially separated by a period ratio larger than two, convergent migration will most likely lead to a very stable 2:1 resonance. Rapid type III migration of the outer planet crossing the 2:1 resonance is one possible way around this problem. In this paper, we investigate this idea in detail. We present an estimate of the required convergent migration rate and confirm this with $\mathrm{N}$-body and hydrodynamical simulations. If the dynamical history of the planetary system had a phase of rapid inward migration that forms a resonant configuration, we predict that the orbital parameters of the two planets will always be very similar and thus should show evidence of that. We use the orbital parameters from our simulation to calculate a radial velocity curve and compare it to observations. Our model provides a fit that is as good as the previously reported one. The eccentricities of both planets are considerably smaller and the libration pattern is different. Within a few years, it will be possible to observe the planet-planet interaction directly and thus distinguish between these different dynamical states.
\end{abstract}

Key words. planets ans satellites: formation - celestial mechanics - protoplanetary disks - accretion, accretion disks

\section{Introduction}

Over 400 extrasolar planets have already been discovered ${ }^{1}$ and their diversity keeps challenging planet formation theory. For example, the recently discovered multi-planetary system HD 45364 raises interesting questions about its formation history.

The planets have masses of $m_{1}=0.1906 M_{\text {Jup }}$ and $m_{2}=$ $0.6891 M_{\text {Jup }}$ and are orbiting the star at a distance of $a_{1}=$ $0.6813 \mathrm{AU}$ and $a_{2}=0.8972 \mathrm{AU}$, respectively (Correia et al. 2009). The period ratio is close to 1.5 , and a stability analysis implies that the planets are deep inside a 3:2 mean motion resonance. The planets have most likely formed further out in cooler regions of the proto-stellar disc as water ice, which is an important ingredient for dust aggregation can only exist beyond the snow line, which is generally assumed to be at radii larger than 2 AU (Sasselov \& Lecar 2000).

It is then usually assumed that migration due to planet disc interactions has moved the planets closer to the star. Although the details of this process are still being hotly debated, the existence of many resonant multiplanetary systems supports this idea. During migration the planets can get locked into a commensurability, after which the planets migrate together with a constant orbital period ratio. In such a resonance, one or more resonant angles are librating (see e.g. Lee \& Peale 2001).

For the planetary system HD 45364, this standard picture poses a new problem. Assuming that the planets have formed far apart, the outcome for the observed masses after migration is almost always a 2:1 mean motion resonance, not 3:2 as observed. The $2: 1$ resonance that forms is found to be extremely

${ }^{1}$ Schneider, J. (2009), http: //exoplanet.eu stable. One possible way around this is a very rapid convergent migration phase that passes quickly through the $2: 1$ resonance.

In this paper we explore this idea quantitatively. The plan of the paper is as follows. In Sect. 2 we use $N$-body simulations to show that the system always ends up in the 2:1 resonance when assuming moderate migration rates. We present scenarios that result in formation of a 3:2 resonance after a rapid migration phase. In Sect. 3 we perform hydrodynamic simulations with a variety of disc models in order to explore the dependence on the physical setup. In Sect. 4 we briefly discuss other formation scenarios. We go on to compare the orbital parameters of our simulations with the observed radial velocity data in Sect. 5 .

We find that the orbital parameters observed in our simulations differ from those estimated from a statistical fit by Correia et al. (2009). However, our models reproduce the observational data very well and we argue that our fit has the same level of significance as that obtained by Correia et al. (2009).

Future observations will be able to resolve this issue. This is the first prediction of orbital parameters for a specific extrasolar planetary system derived from planet migration theory alone. The parameter space of orbital configurations produced by planet disc interactions (low eccentricities, relatively small libration amplitudes) is very small. As in the case of the GJ876 system, this can provide strong evidence of how the system formed. Finally, we summarise our results in Sect. 6.

\section{Formation of HD 45364}

\subsection{Convergent migration and resonance capture}

In the core accretion model (for a review see e.g. Lissauer 1993), a solid core is firstly formed by dust aggregation. This process 
is much more efficient if water exists in a solid form. In the protostellar nebula, this happens beyond the ice line where the temperature is below $150 \mathrm{~K}$ at distances greater than a few AU. Subsequently, after a critical mass is attained (Mizuno 1980), the core accretes a gaseous envelope from the nebula (Bodenheimer \& Pollack 1986). Both planets in the HD 45364 system are inside to the ice line, implying that they should have migrated inwards.

The migration rate depends on many parameters of the disc such as surface density, viscosity, and the mass of the planets. The planets are therefore in general expected to have different migration rates, which leads to the possibility of convergent migration. In this process the planets approach orbital commensurabilities. If they do this slowly enough, resonance capture may occur (Goldreich 1965), after which they migrate together maintaining a constant period ratio thereafter.

Studies made by several authors have shown that when two planets, either of equal mass or with the outer one more massive, undergo differential convergent migration, capture into a mean motion commensurability is expected provided that the convergent migration rate is not too fast (Snellgrove et al. 2001). The observed inner and outer planet masses are such that, if (as is commonly assumed for multiplanetary systems of this kind) the planets are initially separated widely enough that their period ratio exceeds 2, a 2:1 commensurability is expected to form at low migration rates (e.g. Nelson \& Papaloizou 2002; Kley et al. 2004).

Pierens \& Nelson (2008) have studied a similar scenario where the goal was to resemble the $3: 2$ resonance between Jupiter and Saturn in the early solar system. They also find that the 2:1 resonance forms in early stages; however, in their case the inner planet had the higher mass, whereas the planetary system that we are considering has the heavier planet outside. In their situation the 2:1 resonance can be unstable, enabling the formation of a 3:2 resonance later on, and the migration rate may stall or even reverse (Masset \& Snellgrove 2001).

\subsection{The 2:1 mean motion resonance}

We found that, if two planets with masses of the observed system are in a 2:1 mean motion resonance, which has been formed via convergent migration, this resonance is very stable. An important constraint arises, because at the slowest migration rates a 2:1 resonance is expected to form rather than the $3: 2$ commensurability that is actually observed provided the planets start migrating outside any low-order commensurability.

We can estimate the critical relative migration timescale $\tau_{a \text {,crit }}$ above which a 2:1 commensurability forms with the condition that the planets spend at least one libration period migrating through the resonance. The resonance's semi-major axis width $\Delta a$ associated with the $2: 1$ resonance can be estimated from the condition that two thirds of the mean motion difference across $\Delta a$ be equal in magnitude to $2 \pi$ over the libration period. This gives

$\Delta a=\frac{\omega_{l f} a_{2}}{n_{2}}$

where $a_{2}$ and $n_{2}$ are the semi major axis and the mean motion of the outer planet, respectively. The libration period $2 \pi / w_{l f}$ can be expressed in terms of the orbital parameters (see e.g. Goldreich 1965; Rein \& Papaloizou 2009) but is, for convenience, here measured numerically. If we assume the semi-major axes of the two planets evolve on constant (but different) timescales $\left|a_{1} / \dot{a}_{1}\right|=\tau_{a, 1}$ and $\left|a_{2} / \dot{a}_{2}\right|=\tau_{a, 2}$, the condition that the resonance

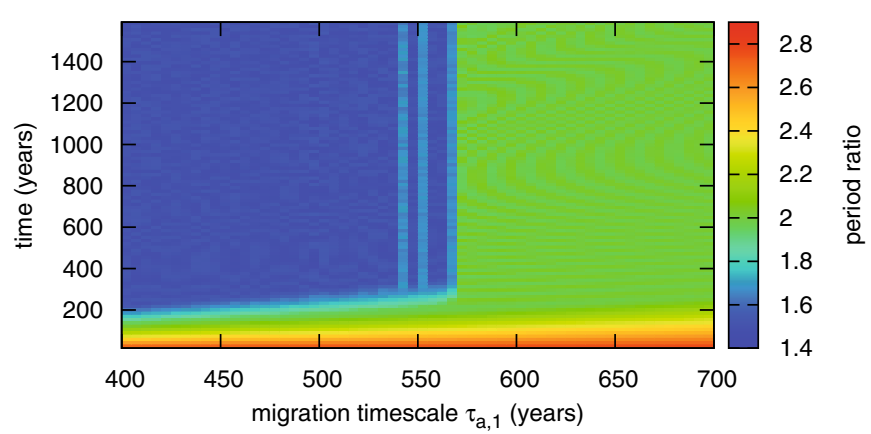

Fig. 1. Period ratio $P_{2} / P_{1}$ as a function of time ( $y$-axis) and migration timescale of the outer planet $\tau_{a, 2}$ ( $y$-axis). The migration timescale of the inner planet is $\tau_{a, 1}=2000$ yrs. The inner planet is initially placed at $r_{1}=1 \mathrm{AU}$. The eccentricity damping is given through $K \equiv \tau_{a} / \tau_{e}=10$.

width is not crossed within a libration period gives

$\tau_{a, \text { crit }} \equiv\left|\frac{1}{1 / \tau_{a, 1}-1 / \tau_{a, 2}}\right| \gtrsim 2 \pi \frac{a_{2}}{\omega_{l f} \Delta a}=2 \pi \frac{n_{2}}{\omega_{l f}^{2}}$

to pass through the $2: 1 \mathrm{MMR}$.

If the planets of the HD45364 system are placed in a 2:1 resonance with the inner planet located at $1 \mathrm{AU}$, the libration pe$\operatorname{riod} 2 \pi / \omega_{l f}$ is found to be approximately $75 \mathrm{yrs}$. Thus, a relative migration timescale shorter than $\tau_{a \text {,crit }} \approx 810 \mathrm{yrs}$ is needed to pass through the $2: 1$ resonance. For example, if we assume that the inner planet migrates on a timescale of 2000 years, the outer planet has to migrate with a timescale of

$\tau_{a, 2, \text { crit }} \lesssim 576$ yrs.

We ran several $N$-body simulations to explore the large parameter space and confirm the above estimate. The code used is similar to the one presented in Rein \& Papaloizou (2009) and uses a fifth order Runge-Kutta, as well as a Burlish Stoer integrator, both with adaptive time-stepping. Different modules deal with migration and stochastic forcing. Non conservative forces are calculated according to the procedure presented in Lee \& Peale (2002) where the migration and eccentricity damping timescales $\tau_{a}=|a / \dot{a}|$ and $\tau_{e}=|e / \dot{e}|$ are imposed for each planet individually. Stochastic forcing was employed only for one run in this paper and we refer the reader to Rein \& Papaloizou (2009) for more details on the implementation.

We place both planets on circular orbits at $a_{1}=1 \mathrm{AU}$ and $a_{2}=2$ AU initially. The migration timescale for the inner planet is fixed at $\tau_{a, 1}=2000 \mathrm{yrs}$, while the migration timescale for the outer planet is varied. In Fig. 1 we plot the period ratio $P_{2} / P_{1}$ as a function of time $t$ for different migration timescales $\tau_{a, 2}$. There is a sharp transition of the final resonant configuration from $2: 1$ to $3: 2$ at around $\tau_{a, 2} \approx 565$ yrs. This value agrees closely with the analytic estimate.

The above results show that, if the planets begin with a period ratio exceeding two, to get them into the observed $3: 2$ resonance, the relative migration time has to be shorter than what is obtained from the standard theory of type II migration applied to these planets in a standard model disc (Nelson et al. 2000). In that case one expects this timescale to be $\sim 10^{4} \mathrm{yrs}$. However, it is possible to obtain the required shorter migration timescales in a massive disc in which the planets migrate in a type III regime (see e.g. Masset \& Papaloizou 2003; Pepliński et al. 2008). In this regime, the surface density distribution in the co-orbital region is asymmetric, leading to a large torque able to cause the planet to fall inwards on a much shorter timescale 
than the disc evolution time obtained for type II migration. We explore this possibility in more detail in the next section.

\section{Hydrodynamical simulations}

Two-dimensional, grid-based hydrodynamic simulations of two gravitationally interacting planets that also undergo interaction with an accretion disc were performed to test the rapid migration hypothesis. The simulations performed here are similar in concept to those performed by Snellgrove et al. (2001) of the resonant coupling in the GJ876 system that may have been induced by orbital migration resulting from interaction with the protoplanetary disc. We performed studies using the FARGO code (Masset 2000) with a modified locally isothermal equation of state. Those runs are indicated by the letter $\mathrm{F}$.

We also ran simulations including viscous heating and radiative transport using the RH2D code, very similar to Kley \& Crida (2008). Those runs are indicated by the letter R.

\subsection{Equation of state}

The outer planet is likely to undergo rapid type III migration, and the co-orbital region will be very asymmetric. We found, in accordance with Pepliński et al. (2008), that the standard softening description does not lead to convergent results in this case (for a comparative study see Crida et al. 2009). Because of the massive disc, a high density spike develops near the planet. Any small asymmetry will then generate a large torque, leading to erratic results. We follow the prescription of Pepliński et al. (2008) and increase the sound speed near the outer planet since the locally isothermal model breaks down in the circumplanetary disc. The new sound speed is given by

$c_{\mathrm{s}}=\left[\left(h_{\mathrm{s}} r_{\mathrm{s}}\right)^{-n}+\left(h_{\mathrm{p}} r_{\mathrm{p}}\right)^{-n}\right]^{-1 / n} \cdot \sqrt{\Omega_{\mathrm{s}}^{2}+\Omega_{\mathrm{p}}^{2}}$

where $r_{\mathrm{s}}$ and $r_{\mathrm{p}}$ are the distance to the star and the outer planet, respectively. Both $\Omega_{\mathrm{s}}$ and $\Omega_{\mathrm{p}}$ are the Keplerian angular velocity and $h_{\mathrm{s}}$ and $h_{\mathrm{p}}$ are the aspect ratio, both of the circumstellar and circumplanetary disc, respectively. The parameter $n$ is chosen to be 3.5 , and the aspect ratio of the circumplanetary disc is $h_{\mathrm{p}}=0.4$. The sound speed has not been changed in the vicinity of the inner, less massive planet as it will not undergo type III migration, and the density peak near the planet is much lower.

\subsection{Radiation hydrodynamic simulations}

In the radiative simulations, we go beyond the locally isothermal approximation and include the full thermal energy equation, which takes the generated viscous heat and radiative transport into account,

$$
\frac{\partial\left(\Sigma c_{\mathrm{v}} T\right)}{\partial t}+\nabla \cdot\left(\Sigma c_{\mathrm{v}} T \boldsymbol{u}\right)=-p \nabla \cdot \boldsymbol{u}+D-Q-2 H \nabla \cdot \boldsymbol{F},
$$

where $\boldsymbol{u}=\left(u_{r}, u_{\varphi}\right)$ is the two-dimensional velocity, $\Sigma$ the surface density, $p$ the (vertically averaged) pressure, $T$ the (mid-plane) temperature of the disc, and $c_{\mathrm{v}}$ the specific heat at constant volume. On the righthand side, the first term describes compressional heating, $D$ the (vertically averaged) dissipation function, $Q$ the local radiative cooling from the two surfaces of the disc $\left(\propto T_{\mathrm{eff}}^{4}\right)$, and $\boldsymbol{F}$ denotes the two-dimensional radiative flux in the $(r, \varphi)$-plane. We calculate the effective temperature of the disc by $T_{\text {eff }}^{4}=\tau T^{4}$, where $\tau$ is the vertical optical depth of the disc. To calculate $\tau$, we use a vertically averaged opacity.
For numerical stability we integrate the diffusion part implicitly. More details about solving the energy equation are stated in Kley \& Crida (2008), and the code RH2D is described in more detail in Kley (1999). In this radiative formulation, the sound speed is a direct outcome of the simulations so we do not use Eq. (4).

\subsection{Initial configuration and computational set up}

We use a system of units in which the unit of mass is the central mass $M_{*}$, the unit of distance is the initial semi-major axis of the inner planet, $r_{1}$, and the unit of time is $\left(G M_{*} / r_{1}^{3}\right)^{-1 / 2}$, thus the orbital period of the initial orbit of the inner planet is $2 \pi$ in these dimensionless units. The parameters for some of the simulations we conducted, as well as their outcomes, are given in Table 1. The first column labels the run, the second and the third columns give the mass ratios relative to the central mass of the inner and outer planets, respectively. The fourth column gives the initial ratio of the semi-major axes of the two planets. The fifth column gives the initial disc aspect ratio, the sixth the softening length in units of the local scale height, the seventh the constant dimensionless viscosity and the eighth column gives the initial surface density. The ninth and tenth columns give the inner and outer boundary of the $2 \mathrm{D}$ grid, respectively. The eleventh and twelfth columns give the radial and azimuthal grid resolutions used and finally the simulation outcome is indicated in the final column.

In all simulations, a smoothing length $b=0.6 H$ has been adopted, where $H$ is the disc thickness at the planet's position. This corresponds to about 4 zone widths in a simulation with a resolution of $768 \times 768$. The role of the softening parameter $b$ that is used in two-dimensional calculations is to account for the smoothing that would result from the vertical structure of the disc in three dimensional calculations (e.g. Masset et al. 2006). We find that the migration rate of the outer planet is independent of the smoothing length if the sound speed is given by Eq. (4).

The planets are initialised on circular orbits. The mass ratios adopted are those estimated for HD 45364. We assume there is no mass accretion, so that these remain fixed in the simulation (see discussion in Pepliński et al. 2008, and also Sect. 4). The initial surface density profile is constant, and tests indicate that varying the initial surface density profile does not change the outcome very much. Non-reflecting boundary conditions have been used throughout this paper.

\subsection{Simulation results}

We find several possible simulation outcomes. These are indicated in the final column of Table 1, which gives the resonance obtained in each case with a final letter D denoting that the migration became ultimately divergent, resulting in the loss of commensurability. We find that convergent migration could lead to a 2:1 resonance that was set up in the initial stages of the simulation (F3). Cases providing more rapid and consistent convergent migration than $\mathrm{F} 3$ could attain a $3: 2$ resonance directly $(\mathrm{F} 1, \mathrm{~F} 2, \mathrm{~F} 4, \mathrm{~F} 5)$.

Thus avoidance of the attainment of sustained $2: 1$ commensurability and the effective attainment of 3:2 commensurability required a rapid convergent migration, as predicted by the $N$-body simulations and the analytic estimate (see Sect. 2.2). That is apparently helped initially by a rapid inward migration phase of the outer planet that approaches a type III migration. The outer planet went through that phase in all simulations with a surface density higher than 0.00025 , and a 3:2 commensurability was obtained. Thus, a surface density comparable to the 
Table 1. Parameters for some of the hydrodynamic simulations.

\begin{tabular}{l|lll|llll|llll|l}
\hline \hline Run & $10^{4} q_{1}$ & $10^{4} q_{2}$ & $r_{2} / r_{1}$ & $H / R$ & $b / H$ & $v$ & $\Sigma$ & $r_{i}$ & $r_{o}$ & $N_{r}$ & $N_{\phi}$ & Result \\
\hline F1 & 2.18 & 7.89 & 1.7 & 0.05 & 0.6 & $10^{-5}$ & 0.001 & 0.25 & 3.0 & 768 & 768 & $3: 2$ \\
F2 & 2.18 & 7.89 & 1.7 & 0.05 & 0.6 & $10^{-5}$ & 0.0005 & 0.25 & 3.0 & 768 & 768 & $3: 2 \mathrm{D}$ \\
F3 & 2.18 & 7.89 & 1.7 & 0.05 & 0.6 & $10^{-5}$ & 0.00025 & 0.25 & 3.0 & 768 & 768 & $2: 1 \mathrm{D}$ \\
F4 & 2.18 & 7.89 & 1.7 & 0.04 & 0.6 & $10^{-5}$ & 0.001 & 0.25 & 3.0 & 768 & 768 & $3: 2 \mathrm{D}$ \\
F5 & 2.18 & 7.89 & 1.7 & 0.07 & 0.6 & $10^{-5}$ & 0.001 & 0.25 & 3.0 & 768 & 768 & $3: 2$ \\
R1 & 2.18 & 7.89 & 1.7 & $\mathrm{rad}$ & 1.0 & $10^{-5}$ & 0.0005 & 0.25 & 3.0 & 300 & 300 & $3: 2$ \\
\hline
\end{tabular}

See Sect. 3.3 for a description.

minimum solar nebula (MMSN, Hayashi 1981), as used in simulation F3, is not sufficient to allow the planets undergo rapid enough type III migration. As soon as the planets approach the 3:2 commensurability, type III migration stops due to the interaction with the inner planet and the outer planet starts to migrate in a standard type II regime.

This imposes another constraint on the long-term sustainability of the resonance. The inner planet remains embedded in the disc and thus potentially undergoes a fairly rapid inward type I migration. If the type II migration rate of the outer planet is slower than the type I migration rate of the inner planet, then the planets diverge and the resonance is not sustained. A a precise estimate of the migration rates is impossible in late stages, as the planets interact strongly with the density structure imposed on the disc by each other.

Accordingly, outflow boundary conditions at the inner boundary that prevent the build up of an inner disc are more favourable to the maintenance of a 3:2 commensurability because the type I migration rate scales linearly with the disc surface density. However, those are not presented here, as the effect is weak and we stop the simulation before a large inner disc can build up near the boundary.

The migration rate for the inner planet depends on the aspect ratio $h$. It is decreased for an increased disc thickness (Tanaka et al. 2002). That explains why models with a large disc thickness (F5) tend to stay in resonance, whereas models with a smaller disc thickness (F2, F4) tend towards divergent migration at late times. The larger thickness is consistent with radiative runs (see below).

As an illustration of the evolution from a typical configuration (F5) that forms and maintains a 3:2 commensurability during which the orbital radii contract by a factor of at least $\sim 2$ we plot the evolutions of the semi-major axes, the period ratio, and eccentricities in Fig. 2 and provide a surface density contour plot in Fig. 3 after 100 initial inner planet orbits at which the 3:2 commensurability has just been established.

The eccentricity peak in Fig. 2 at $t \sim 300$ comes from passing through the 2:1 commensurability. At $t \sim 800$, the 3:2 commensurability is reached and maintained until the end of the simulation. The surface density in this simulation is approximately 5 times higher than the MMSN at 1 AU (Hayashi 1981).

We also present an illustration of the evolution of the semi major axes and eccentricities, as well as a surface density plot from a simulation (F4) that does form a 3:2 commensurability, but loses it because the inner planet is migrating too fast in Figs. 4 and 5. One can see that a massive inner disc has piled up. This and the small aspect ratio of $h=0.04$ make the inner planet go faster than the outer planet, which has opened a clear gap. The commensurability is lost at $t \sim 1200$.

The radiative run $R 1$ also shows capture in a 3:2 resonance which is sustained. Before embedding the planets, the disc is first brought into radiative equilibrium where viscous heating is balanced by radiative cooling. During this evolution, the surface density is kept constant as in the local isothermal models. The temperature of the disc is obtained directly from the model, and the equilibrium is equivalent to approximately $H / r=0.075$ at one AU. The planets are then embedded at the same initial positions as in the other models (F1-F5). We plot the evolution of the semi major axes, period ratio and eccentricities in Fig. 6. When reaching the location of the 2:1 resonance, the eccentricities of both planets increase initially but decline subsequently after the resonance is transversed. Capture into 3:2 occurs smoothly during the inward migration of the planets. The final eccentricities reached in this model are around $e_{1}=0.03$ and $e_{2}=0.01$.

The initial type III migration rate is slower when compared to run F5 because the surface density is lower. After the the 2:1 resonance is passed, the outer planet migrates in a type II regime, so that the capture into the $3: 2$ resonance appears later. However, the orbital parameters measured at the end of the simulations are very similar to any other run that we performed. This indicates that the parameter space that is populated by this kind of planet disc simulation is very generic.

We tested the effect of disc dispersal at the late stages in our models to evolve the system self-consistently to the present day. In model $\mathrm{F} 5$ after $t=2000$ we allow the disc mass to exponentially decay on a timescale of $\tau_{\text {dis }} \sim 2000$. This timescale is shorter than the photo-evaporation timescale (Alexander et al. 2006). However, this scenario is expected to give a stronger effect than a long timescale (Sándor \& Kley 2006). In agreement with those authors, we found that the dynamical state of the system does not change for the above parameters. At a late stage, the resonance is well established and the planets undergo a slow inward migration. Strong effects are only expected if the disc dispersal happens during the short period of rapid type III migration, which is very unlikely. We observed that the eccentricities show a trend toward decreasing and the libration amplitudes tend toward slightly increasing during the dispersal phase. However, these changes are not different than what has been observed in runs without a disappearing disc.

\section{Other scenarios for the origin of HD45364}

In the above discussion, we have considered the situation when the planets attain their final masses while having a wider separation than required for a 2:1 commensurability, and found that convergent migration scenarios can be found that bring them into the observed 3:2 commensurability by disc planet interactions. However, it is possible that they could be brought to their current configuration in a number of different ways as considered below. It is important to note that, because the final commensurable state results from disc planet interactions, it should have 

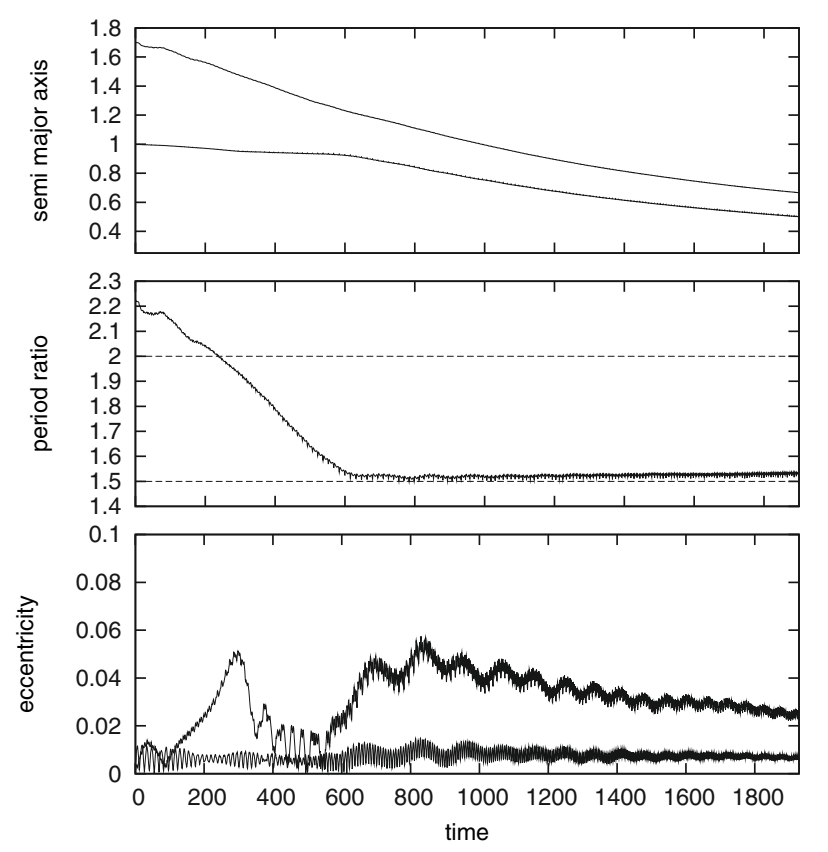

Fig. 2. The semi-major axes (top), period ratio $P_{2} / P_{1}$ (middle), and eccentricities (bottom) of the two planets plotted as a function of time in dimensionless units for run F5 with a disc aspect ratio of $h=0.07$. In the bottom panel, the upper curve corresponds to the inner planet.

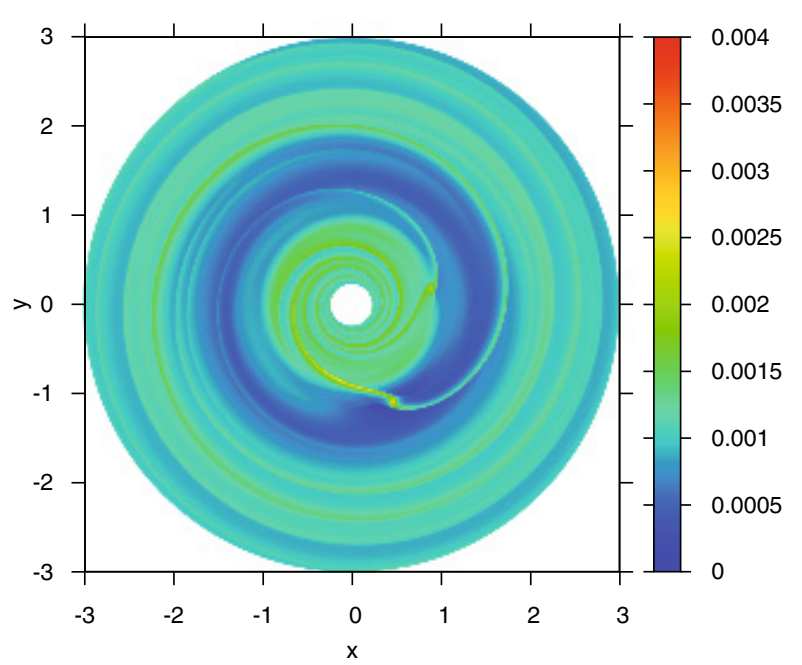

Fig. 3. A surface-density contour plot for simulation F 5 after 100 orbits at the end of the type III migration phase. The outer planet establishes a definite gap, while the inner planet remains embedded at the edge of the outer planet's gap.

similar properties to those described above when making comparisons with observations.

It is possible that the solid cores of either both planets or just the outer planet approached the inner planet more closely than the $2: 1$ commensurability before entering the rapid gas accretion phase and attaining their final masses prior to entering the $3: 2$ commensurability. Although it is difficult to rule out such possibilities entirely, we note that the cores would be expected to be in the super earth mass range, where in general closer commensurabilities than $2: 1$ and even 3:2 are found for typical type I migration rates (e.g. Papaloizou \& Szuszkiewicz 2005; Cresswell \& Nelson 2008). One may also envisage the possibility that the solid cores grew in situ in a 3:2 commensurability, but this would have to survive expected strongly varying migration
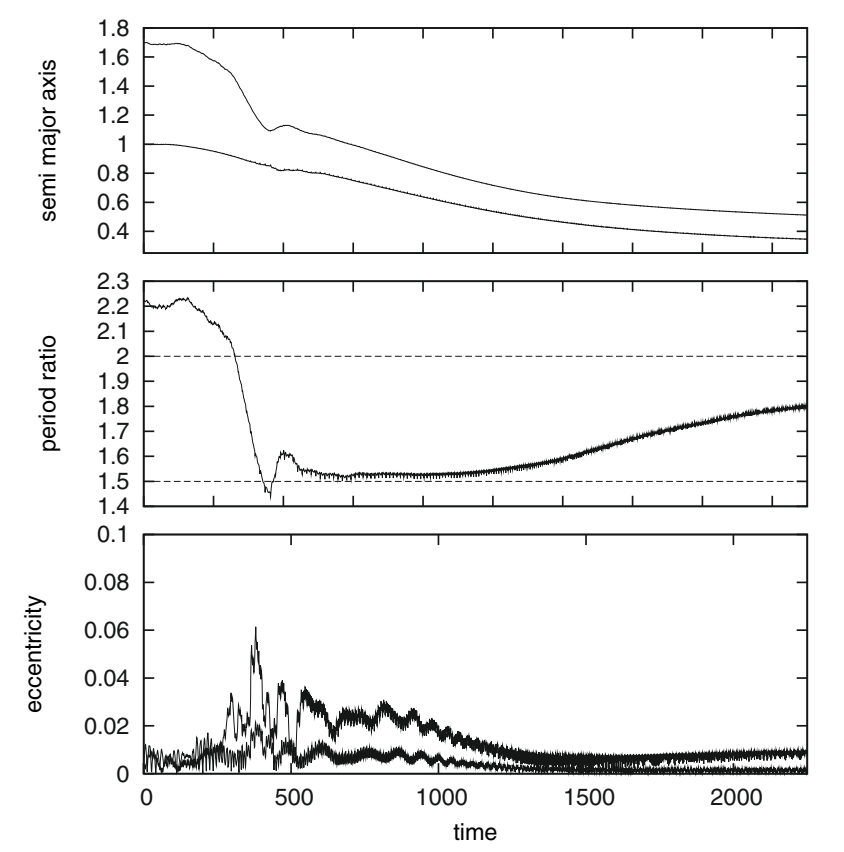

Fig. 4. The semi-major axes (top), period ratio $P_{2} / P_{1}$ (middle), and eccentricities (bottom) of the two planets plotted as a function of time in dimensionless units for run F4 with a disc aspect ratio of $h=0.04$. In the bottom panel, the upper curve corresponds to the inner planet.

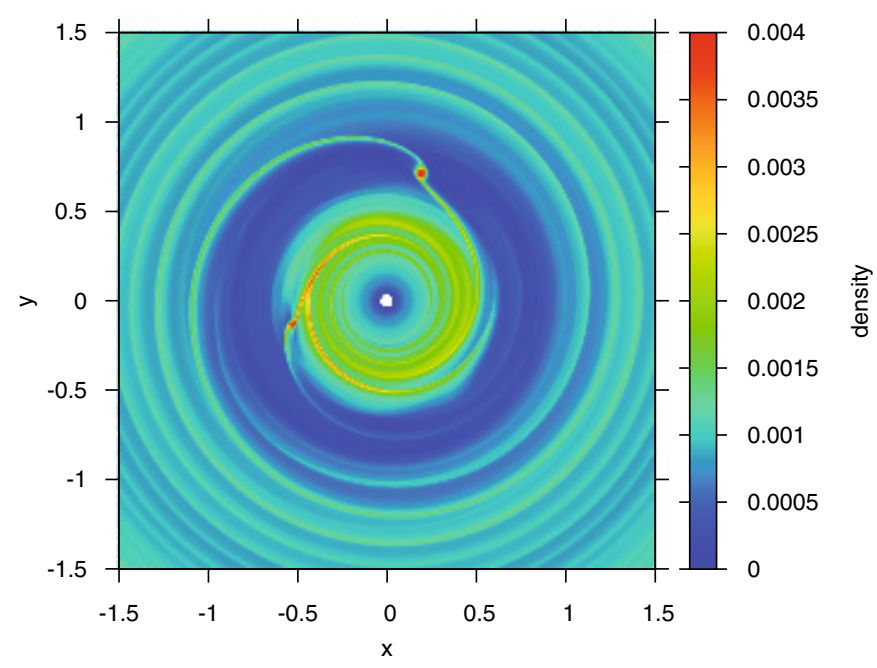

Fig. 5. A surface-density contour plot for simulation F4 after 150 orbits after the planets went into divergent migration. The inner planet is embedded and interacts strongly with the inner disc. The simulation uses a 1 D grid for $0.04<r<0.25$.

rates as a result of disc planet interactions as the planets grew in mass.

An issue is whether the embedded inner planet is in a rapid accretion phase. The onset of the rapid accretion phase (also called phase 3 ) occurs when the core and envelope mass are about equal (Pollack et al. 1996). The total planet mass depends at this stage on the boundary conditions, here determined by the circumplanetary flow. When these allow the planet to have a significant convective envelope, the transition to rapid accretion may not occur until the planet mass exceeds $60 M_{\oplus}$ (Wuchterl 1993), which is the mass of the inner planet (see also model J3 of Pollack et al. 1996; and models of Papaloizou \& Terquem 1999). Because of the above results, it is reasonable that the inner planet is not in a rapid accretion phase. 
A\&A 510, A4 (2010)
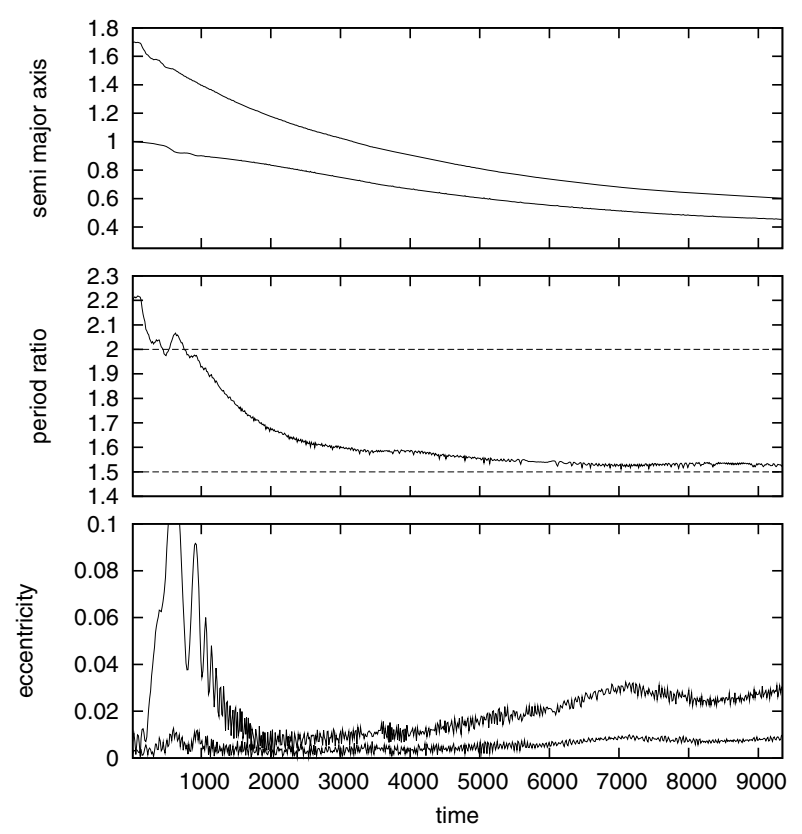

Fig. 6. The semi-major axes (top), period ratio $P_{2} / P_{1}$ (middle) and eccentricities (bottom) of the two planets plotted as a function of time in dimensionless units for the radiative run R1.

Finally we remark that protoplanetary discs are believed to maintain turbulence in some part of their structure. Using the prescription of Rein \& Papaloizou (2009), we can simulate the turbulent behaviour of the disc by adding additional forces to an $\mathrm{N}$-body simulation. These forces will ultimately eject the planets from the 2:1 resonance should that form. Provided they are strong enough, this can happen within the lifetime of the disc, thus making a subsequent capture into the observed 3:2 resonance possible.

We confirmed numerically that such cases can occur for moderately large diffusion coefficients (as estimated by Rein \& Papaloizou 2009). However, this outcome seems to be the exception rather than the rule. Should the 2:1 resonance be broken, a planet-planet scattering event appears to be more likely. In all the simulations we performed, we find that only a small fraction of systems (1-5\%) eventually end up in a 3:2 resonance.

\section{Comparison with observations}

Table 2 lists the orbital parameters that Correia et al. (2009) obtained from their best statistical fit of two Keplerian orbits to the radial velocity data. The radial velocity data collected so far is insufficient for detecting any interactions between the planets. This is borne out by the fact that the authors did not obtain any improvement in terms of minimising $\chi^{2}$ when a 3-body Newtonian fit rather than a Keplerian fit was carried out. However, a stability analysis supports the viability of the determined parameters, as these lie inside a stable region of orbital parameter space. The best-fit solution shows a 3:2 mean motion commensurability, although no planet-planet interactions have been observed from which this could be inferred directly. It is important to note that the large minimum $\chi^{2}$ associated with the best-fit that could be obtained indicates large uncertainties that are not accounted for by the magnitudes of the errors quoted in their paper.

There are two main reasons we believe that the effective errors associated with the observations are large. First, many data
Table 2. Orbital parameters of HD 45364b and HD 45364c from different fits.

\begin{tabular}{|c|c|c|c|}
\hline & & Correia et al. (2009) & Simulation F5 \\
\hline Parameter & Unit & $\mathrm{b} \quad \mathrm{c}$ & $\mathrm{b} \quad \mathrm{c}$ \\
\hline$M \sin i$ & {$\left[M_{\text {Jup }}\right]$} & 0.1872 & $\begin{array}{ll}0.1872 & 0.6579\end{array}$ \\
\hline$M_{*}$ & {$\left[M_{\odot}\right]$} & 0.82 & 0.82 \\
\hline$a$ & [AU] & 0.8972 & $0.6804 \quad 0.8994$ \\
\hline$e$ & & $0.17 \pm 0.02 \quad 0.097 \pm 0.012$ & 0.036 \\
\hline$\lambda$ & [deg] & $105.8 \pm 1.4 \quad 269.5 \pm 0.6$ & 352.5 \\
\hline$\varpi^{a}$ & [deg] & $162.6 \pm 6.3 \quad 7.4 \pm 4.3$ & 292.2 \\
\hline$\sqrt{x^{2}}$ & & 2.79 & $2.76^{b}(3.51)$ \\
\hline Date & [JD] & 2453500 & 2453500 \\
\hline
\end{tabular}

Notes. ${ }^{(a)}$ Note that $\varpi$ is not well constrained for nearly circular orbits. (b) radial velocity amplitude was scaled down by $8 \%$.

points are clustered and clearly do not provide a random time sampling. This could result in correlations that effectively reduce the total number of independent measurements. Second, the high minimum $\chi^{2}$ value that is three times the quoted observational error indicates that there are additional effects (e.g. sunspots, additional planets, etc.) that produce a jitter in the central star (Mayor, private communication), which then enhances the effective observational error.

No matter what process generates the additional noise, we can assume in a first approximation that it follows a Gaussian distribution. We then have to conclude that the effective error of each observation is a factor $\sim 3$ larger than reported in order to account for the minimum $\chi^{2}$, given the quoted number of $\sim 60$ independent observations. Under these circumstances, we would then conclude that any fit with $\sqrt{\chi^{2}}$ in the range $2-4$ would be an equally valid possibility.

In the following, we show that indeed a variety of orbital solutions match the observed RV data with no statistically significant difference when compared to the quoted best fit by Correia et al. (2009). As one illustration, we use the simulation F5 obtained in Sect. 3. We take the orbital parameters at a time when the orbital period of the outer planet is closest to the observed value and integrate them for several orbits with our $N$-body code. The solution is stable for at least one million years. There are only two free parameters available to fit the reflex motion of the central star to the observed radial velocity: the origin of time (epoch) and the angle between the line of sight and the pericentre of the planets.

We can assume that the planet masses and periods are measured with high accuracy and that only the shape of the orbit contains large errors. Our best fit results in an unreduced $\sqrt{\chi^{2}}$ value of 3.51. According to the above discussion, this solution is statistically indistinguishable from a solution with $\sqrt{\chi^{2}} \sim 2.8$.

It is possible to reduce the value of $\chi^{2}$ even further, when assuming that the planet masses are not fixed. In that case, we have found a fit corresponding to $\sqrt{\chi^{2}}=2.76$ where we have reduced the radial velocity amplitude by $8 \%$ (effectively adding one free parameter to the fit). This could be explained by either a heavier star, less massive planets, or a less inclined orbit. The orientation of the orbital plane has been kept fixed $\left(i=90^{\circ}\right)$ in all fits.

The results are shown in Fig. 7. The blue curve corresponds to the outcome of simulation F5 and we list the orbital parameters in Table 2. We also plot the best fit given by Correia et al. (2009) for comparison (green curve). In accordance with the discussion given above, it is very difficult to see any differences in 


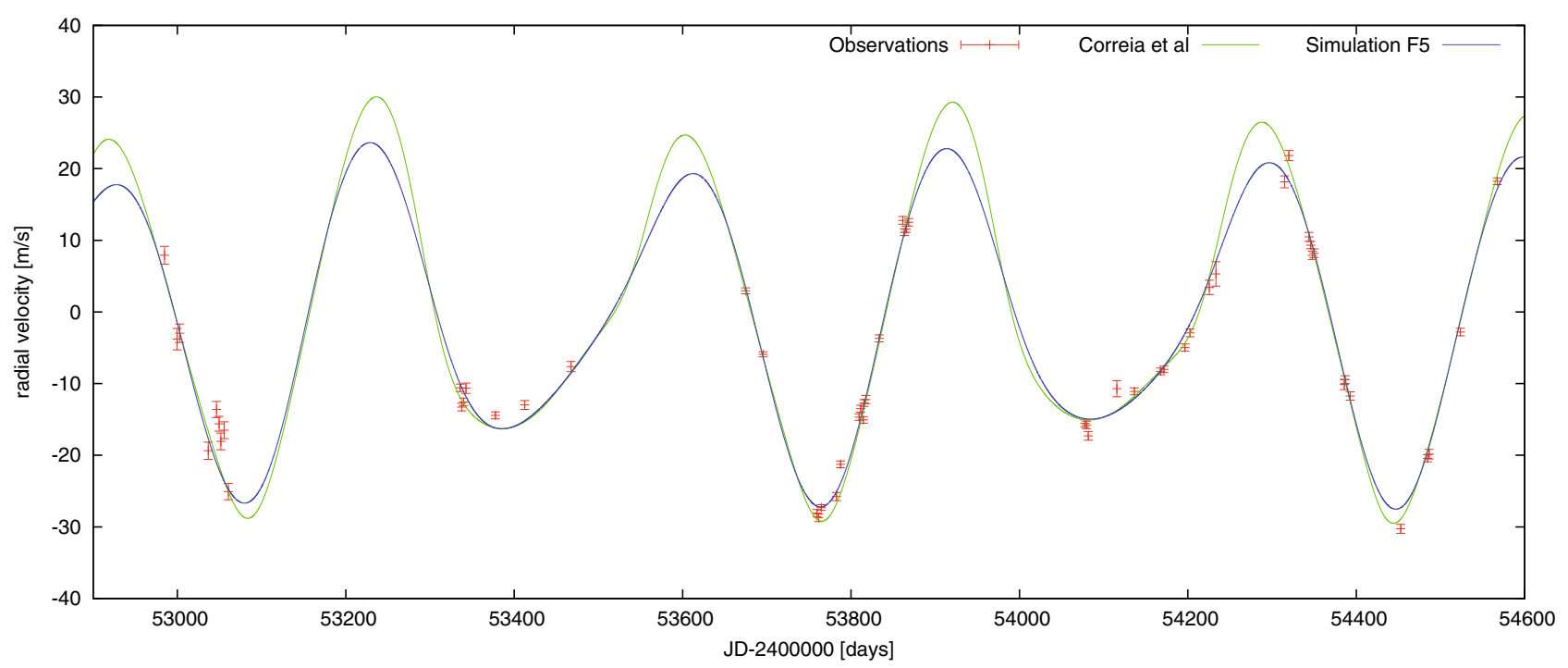

Fig. 7. Comparison of different orbital solutions. See text for a description of the different models. Radial velocity measurements (red points) and the published orbital solution (green curve) are taken from Correia et al. (2009).

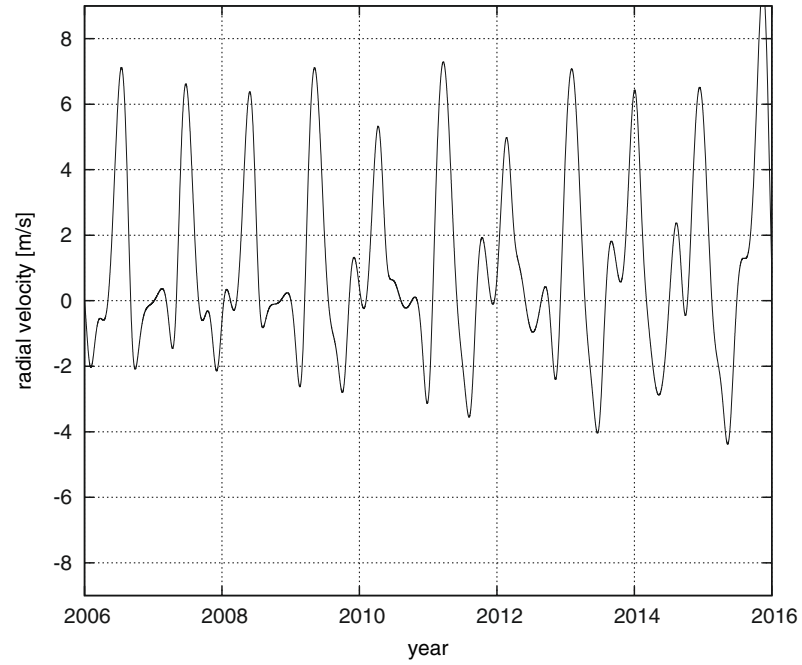

Fig. 8. Difference in radial velocity between the previously reported fit by Correia et al. (2009) and the new fit obtained from simulation F5.

the quality of these fits, which indeed suggests that the models are statistically indistinguishable.

However, there is an important difference between the fits obtained from our simulations and that of Correia et al. (2009). Our models consistently predict lower values for both eccentricities $e_{1}$ and $e_{2}$ (see Table 2). Furthermore, the ratio of eccentricities $e_{1} / e_{2}$ is higher than the previously reported value of 1.73. The eccentricities are oscillating and the ratio is on average $\sim 3$.

This in turn results in a different libration pattern: the slow libration mode (see Fig. 3a in Correia et al. 2009) that is associated with oscillations of the angle between the two apsidal lines is absent, as shown in Fig. 9. Thus there is a marked difference in the form the interaction between the two planets takes in the case of this fit.

It is hoped that future observations will be able to resolve this issue. The evolution of the difference in radial velocity over the next few years between our fit from simulation F5 and the fit found by Correia et al. (2009) is plotted in Fig. 8. There is approximately one window each year that will allow further observations to distinguish between the two models. The large

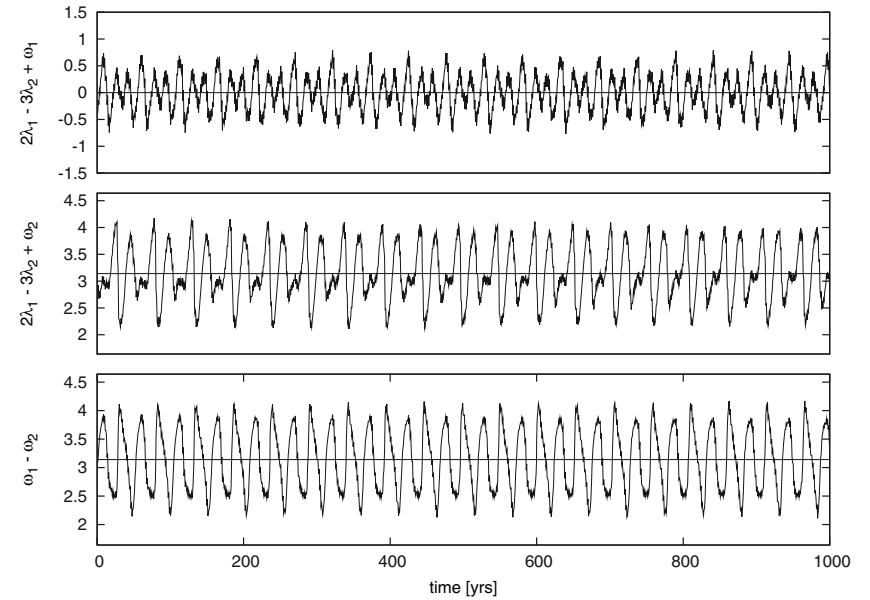

Fig. 9. Evolution of the resonant angles $\phi_{1}=2 \lambda_{1}-3 \lambda_{2}+\varpi_{1}, \phi_{2}=$ $2 \lambda_{1}-3 \lambda_{2}+\varpi_{2}, \varpi_{1}-\varpi_{2}$ for simulation $\mathrm{F} 5$ using the parameters given in Table 2. The angles are measured in radians.

difference at these dates is not caused by to the secular evolution of the system, but is simply a consequence of smaller eccentricities.

All runs that yield a final configuration with a 3:2 MMR are very similar in their dynamical behaviour, as found for model $\mathrm{F} 5$. They are characterised by an antisymmetric $\left(\varpi_{2}-\varpi_{1} \sim \pi\right)$ state with a relatively small libration of $\phi_{1}$ and $\phi_{2}$. This is a generic outcome of convergent disc-planet migration scenarios.

\section{Conclusions}

The planets in the multi-planetary system HD45364 are most likely in a 3:2 mean motion resonance. This poses interesting questions on its formation history. Assuming that the planets form far apart and migrate with a moderate migration rate, as predicted by standard planet formation and migration theories, the most likely outcome is a $2: 1$ mean motion resonance, contrary to the observation of a 3:2 MMR.

In this work, we investigated a possible way around this problem by letting the outer planet undergo a rapid inward 
type III migration. We presented an analytical estimate and performed both $N$-body and hydrodynamical simulations.

We find that it is indeed possible to form a 3:2 MMR and avoid the 2:1 resonance, thus resembling the observed planetary system using reasonable disc parameters. Hydrodynamical simulations suggest that the system is more likely to sustain the resonance for high aspect ratios, as the migration of the inner planet is slowed down, thus avoiding divergent migration.

Finally, we used the orbital configuration found in the hydrodynamical formation scenario to calculate a radial velocity curve. This curve was then compared to observations and the resulting fit has an identical $\chi^{2}$ value to the previously reported best fit.

Our solution is stable for at least a million years. It is in a dynamically different state, both planets having lower eccentricities and a different libration pattern. This is the first time that planet migration theory can predict a precise orbital configuration of a multiplanetary system.

This might also be the first direct evidence for type III migration if this scenario turns out to be true. The system HD 45364 remains an interesting object for observers, as the differences between the two solutions can be measured in radial velocity within a couple of years.

Acknowledgements. The authors are grateful to the Isaac Newton Institute for Mathematical Sciences in Cambridge where the final stages of this work were carried out during the Dynamics of Discs and Planets research programme. Hanno Rein was supported by an Isaac Newton Studentship, the Science and Technology Facilities Council, and St John's College Cambridge. Simulations were performed on the Astrophysical Fluid's Core i7 machines at DAMTP and on Darwin, the Cambridge University HPC facility.

\section{References}

Alexander, R. D., Clarke, C. J., \& Pringle, J. E. 2006, MNRAS, 369, 229 Bodenheimer, P., \& Pollack, J. B. 1986, Icarus, 67, 391

Correia, A. C. M., Udry, S., Mayor, M., et al. 2009, A\&A, 496, 521

Cresswell, P., \& Nelson, R. P. 2008, A\&A, 482, 677

Crida, A., Baruteau, C., Kley, W., \& Masset, F. 2009, A\&A, 502, 679

Goldreich, P. 1965, MNRAS, 130, 159

Hayashi, C. 1981, Progr. Theor. Phys. Suppl., 70, 35

Kley, W. 1999, MNRAS, 303, 696

Kley, W., \& Crida, A. 2008, A\&A, 487, L9

Kley, W., Peitz, J., \& Bryden, G. 2004, A\&A, 414, 735

Lee, M. H., \& Peale, S. J. 2001, in BAAS, 33, 1198

Lee, M. H., \& Peale, S. J. 2002, ApJ, 567, 596

Lissauer, J. J. 1993, ARA\&A, 31, 129

Masset, F. 2000, A\&AS, 141, 165

Masset, F., \& Snellgrove, M. 2001, MNRAS, 320, L55

Masset, F. S., \& Papaloizou, J. C. B. 2003, ApJ, 588, 494

Mizuno, H. 1980, Progr. Theor. Phys., 64, 544

Nelson, R. P., \& Papaloizou, J. C. B. 2002, MNRAS, 333, L26

Nelson, R. P., Papaloizou, J. C. B., Masset, F., \& Kley, W. 2000, MNRAS, 318, 18

Papaloizou, J. C. B., \& Szuszkiewicz, E. 2005, MNRAS, 363, 153

Papaloizou, J. C. B., \& Terquem, C. 1999, ApJ, 521, 823

Pepliński, A., Artymowicz, P., \& Mellema, G. 2008, MNRAS, 386, 164

Pierens, A., \& Nelson, R. P. 2008, A\&A, 482, 333

Pollack, J. B., Hubickyj, O., Bodenheimer, P., et al. 1996, Icarus, 124, 62

Rein, H., \& Papaloizou, J. C. B. 2009, A\&A, 497, 595

Sándor, Z., \& Kley, W. 2006, A\&A, 451, L31

Sasselov, D. D., \& Lecar, M. 2000, ApJ, 528, 995

Snellgrove, M. D., Papaloizou, J. C. B., \& Nelson, R. P. 2001, A\&A, 374, 1092

Tanaka, H., Takeuchi, T., \& Ward, W. R. 2002, ApJ, 565, 1257

Wuchterl, G. 1993, Icarus, 106, 323 